\title{
ARTICLE
}

\section{Evidence for a small hole pocket in the Fermi surface of underdoped $\mathrm{YBa}_{2} \mathrm{Cu}_{3} \mathrm{O}_{y}$}

N. Doiron-Leyraud', S. Badoux², S. René de Cotret', S. Lepault², D. LeBoeuf², F. Laliberté ${ }^{1}$, E. Hassinger ${ }^{1}$,

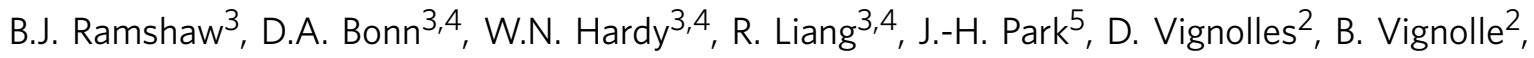
L. Taillefer ${ }^{1,4} \&$ C. Proust ${ }^{2,4}$

In underdoped cuprate superconductors, the Fermi surface undergoes a reconstruction that produces a small electron pocket, but whether there is another, as yet, undetected portion to the Fermi surface is unknown. Establishing the complete topology of the Fermi surface is key to identifying the mechanism responsible for its reconstruction. Here we report evidence for a second Fermi pocket in underdoped $\mathrm{YBa}_{2} \mathrm{Cu}_{3} \mathrm{O}_{y}$, detected as a small quantum oscillation frequency in the thermoelectric response and in the $c$-axis resistance. The field-angle dependence of the frequency shows that it is a distinct Fermi surface, and the normal-state thermopower requires it to be a hole pocket. A Fermi surface consisting of one electron pocket and two hole pockets with the measured areas and masses is consistent with a Fermi-surface reconstruction by the charge-density-wave order observed in $\mathrm{YBa}_{2} \mathrm{Cu}_{3} \mathrm{O}_{y}$ provided other parts of the reconstructed Fermi surface are removed by a separate mechanism, possibly the pseudogap.

\footnotetext{
${ }^{1}$ Département de physique \& RQMP, Université de Sherbrooke, Sherbrooke, Québec, Canada J1K 2R1. ${ }^{2}$ Laboratoire National des Champs Magnétiques Intenses (CNRS, INSA, UJF, UPS), 31400 Toulouse, France. ${ }^{3}$ Department of Physics \& Astronomy, University of British Columbia, Vancouver, British Columbia, Canada V6T 1Z1. ${ }^{4}$ Canadian Institute for Advanced Research, Toronto, Ontario, Canada M5G 1Z8. ${ }^{5}$ National High Magnetic Field Laboratory, Tallahassee, Florida 32310, USA. Correspondence and requests for materials should be addressed to L.T. (email: louis.taillefer@usherbrooke.ca) or to C.P. (email: cyril.proust@Incmi.cnrs.fr).
} 
T he phase diagram of cuprate superconductors is shaped by ordered states, and their identification is essential for understanding high-temperature superconductivity. Evidence for a new state with broken symmetry in cuprates recently came from two major developments. The observation of quantum oscillations in underdoped $\mathrm{YBa}_{2} \mathrm{Cu}_{3} \mathrm{O}_{y}(\mathrm{YBCO})^{1}$ and $\mathrm{HgBa}_{2} \mathrm{CuO}_{4+\mathrm{d}}$ (Hg1201) (ref. 2), combined with negative Hall ${ }^{3-5}$ and Seebeck ${ }^{5-7}$ coefficients, showed that the Fermi surface contains a small closed electron pocket and is therefore reconstructed at low temperature, implying that translational symmetry is broken. The detailed similarity of the Fermi-surface reconstruction in YBCO and $\mathrm{La}_{1.6-}{ }_{-} \mathrm{Eu}_{0.4} \mathrm{Sr}_{x} \mathrm{CuO}_{4}(\mathrm{Eu}-\mathrm{LSCO})^{6-8}$ revealed that YBCO must host a density-wave order similar to the stripe order of Eu-LSCO9. More recently, charge-densitywave $(\mathrm{CDW})$ modulations were observed directly, first by $\mathrm{NMR}$ in $\mathrm{YBCO}^{10}$ and then by X-ray diffraction in $\mathrm{YBCO}^{11-13}$ and Hg1201 (ref. 14). In YBCO, a thermodynamic signature of the CDW order was detected in the sound velocity at low temperature and finite magnetic field ${ }^{15}$. These CDW modulations are reminiscent of the checkerboard pattern previously observed by STM on $\mathrm{Bi}_{2} \mathrm{Sr}_{2} \mathrm{CaCu}_{2} \mathrm{O}_{8+\mathrm{d}}$ (refs 16,17), for instance.

Fermi-surface reconstruction and CDW modulations are therefore two universal signatures of underdoped cuprates, which begs the following question: is the Fermi surface seen by quantum oscillations compatible with a reconstruction by the observed CDW modulations? This issue requires a detailed knowledge of the Fermi surface, to be compared with Fermi surface calculations based on the measured parameters of the CDW order, in the same material at the same doping. In this Article, we report quantum oscillations measurements that reveal an additional, hole-like Fermi pocket in underdoped YBCO. As we discuss below, a Fermi surface consisting of one electron and two hole pockets of the measured sizes and masses is consistent with a reconstruction by the observed CDW.

\section{Results}

We have measured quantum oscillations in the thermoelectric response and $c$-axis resistance of underdoped YBCO. Our samples were chosen to have a doping $p=0.11-0.12$, at which the amplitude of quantum oscillations is maximal ${ }^{18}$. In the doping-temperature phase diagram, this is also where the CDW modulations are strongest ${ }^{19,20}$ (Fig. 1a) and where the critical magnetic field $B_{\mathrm{c} 2}$ needed to suppress superconductivity is at a local minimum ${ }^{21}$ (Fig. 1b). In the $T=0$ limit, the Seebeck $(S)$ and Nernst $(v=N / B)$ coefficients are inversely proportional to the Fermi energy ${ }^{22,23}$ and are therefore expected to be enhanced for small Fermi surfaces. In Fig. 2a, we show isotherms of $S$ and $N$ at $T=2 \mathrm{~K}$ measured up to $B=45 \mathrm{~T}$ in a YBCO sample with $p=0.11$. Above $B_{\mathrm{c} 2}=24 \mathrm{~T}$ (ref. 21), both $S$ and $N$ are negative; the fact that $S<0$ is consistent with an electron pocket dominating the transport at low temperature ${ }^{6,7}$. The normalstate signal displays exceptionally large quantum oscillations, with a main frequency $F_{\mathrm{a}}=540 \mathrm{~T}$ and a beat pattern indicative of other, nearby, frequencies. In Fig. 2, we also show the $c$-axis resistance of two YBCO samples at $p=0.11$ and 0.12 , measured in pulsed fields up to $68 \mathrm{~T}$. The overall behaviour of the $c$-axis magnetoresistance at $p=0.11$ is consistent with previous reports ${ }^{24,25}$. Quantum oscillations are clearly visible and the three distinct frequencies $F_{\mathrm{a} 1}=540 \mathrm{~T}, F_{\mathrm{a} 2}=450 \mathrm{~T}$ and $F_{\mathrm{a} 3}=630 \mathrm{~T}$ in the Fourier spectrum at $p=0.11$ (Fig. 1d) agree with reported values ${ }^{26}$.

With increasing temperature, the amplitude of these 'fast' oscillations decreases rapidly and above $T \sim 10 \mathrm{~K}$ we are left with a slowly undulating normal-state signal, clearly seen in the raw
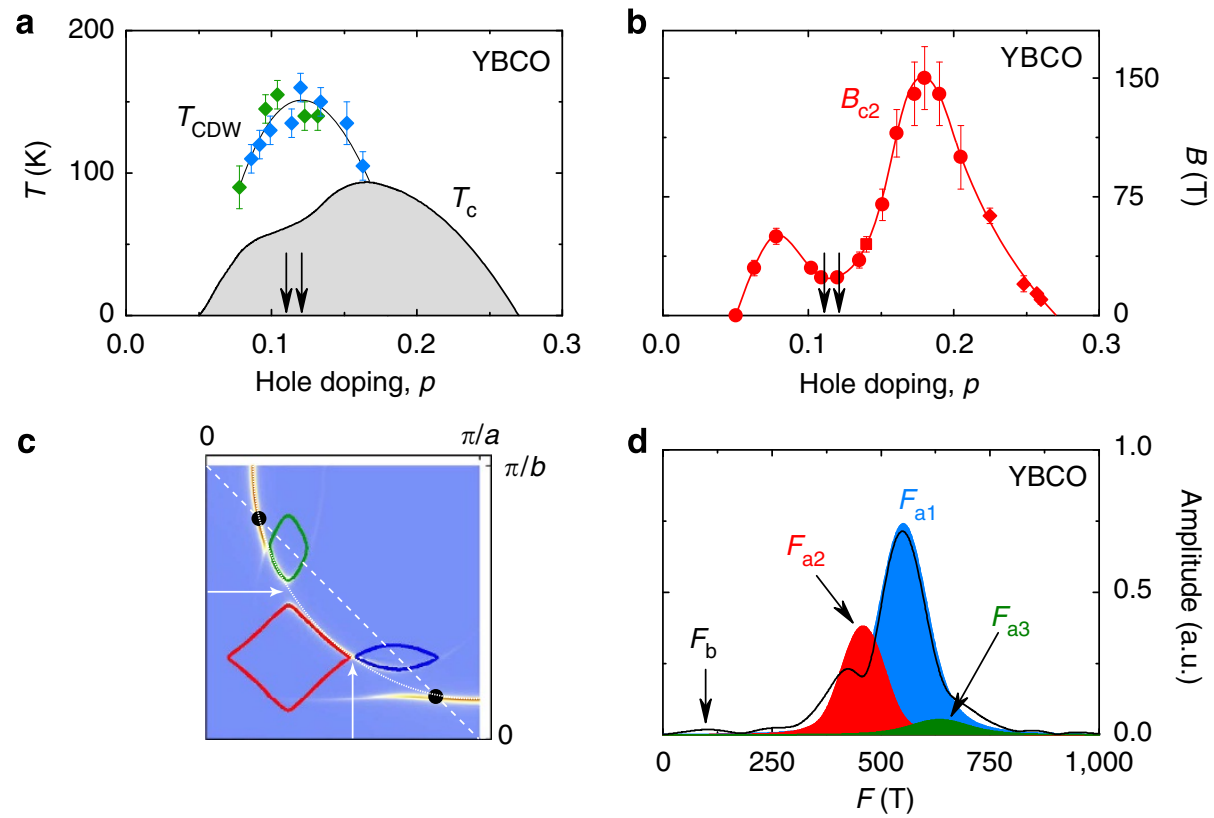

Figure 1 | Phase diagrams and Fermi surface of YBCO. (a) Temperature-doping phase diagram of YBCO, showing the superconducting transition temperature $T_{\mathrm{c}}$ (grey dome, data from ref. 46), the CDW onset temperature $T_{\mathrm{CDW}}$ (green diamonds, from ref. 19; blue diamonds, from ref. 20). (b) Upper critical field $H_{\mathrm{c} 2}$ of $\mathrm{YBCO}$ as a function of doping (red dots, from ref. 21). In both $\mathbf{a}, \mathbf{b}$, the arrows indicate the two dopings of the samples used in our study. All error bars in $\mathbf{a}, \mathbf{b}$ are reproduced from the original references. (c) Sketch of the reconstructed Fermi surface adapted from ref. 42 using the CDW wavevectors (arrows) measured in $\mathrm{YBCO}^{19,20}$, showing a diamond-shaped nodal electron pocket (red) and two hole-like ellipses (blue and green). The dashed line is the antiferromagnetic Brillouin zone; the dotted line is the original large Fermi surface; the black dots mark the so-called 'hot spots', where those two lines intersect. (d) Fourier transform of our c-axis resistance data (at $p=0.11$ ), showing the new 'low' frequency $F_{\mathrm{b}}=95 \pm 10 \mathrm{~T}$ reported here, and the three main 'high' frequencies $F_{\mathrm{a} 1}$ (blue), $F_{\mathrm{a} 2}$ (red) and $F_{\mathrm{a} 3}$ (green). 
Seebeck data (Figs $2 \mathrm{~b}$ and $3 \mathrm{a}$ ). In Fig. 3b, the oscillatory part of that signal, obtained by subtracting a smooth background, is plotted as a function of inverse magnetic field. Although the 'slow oscillations' at $18 \mathrm{~K}$ are 20 times weaker than the fast oscillations at $2 \mathrm{~K}$, they are clearly resolved and periodic in $1 / B$. After their discovery in the Seebeck signal, the slow oscillations were also detected in the $c$-axis resistance, as shown in Fig. $3 c$. In both the Seebeck and $c$-axis resistance data, the frequency of these slow oscillations is $F_{\mathrm{b}}=95 \pm 10 \mathrm{~T}(p=0.11)$. Similar oscillations were also detected in the $c$-axis resistance of a sample at $p=0.12$ (Fig. 3d), with $F_{\mathrm{b}}=120 \pm 15 \mathrm{~T}$. In Fig. $4 \mathrm{a}$, we show the derivative $\mathrm{d} R_{\mathrm{c}} / \mathrm{d} B$, which unambiguously reveals $F_{\mathrm{b}}$, without the need for a background subtraction. (Note that in the $c$-axis resistance data, the amplitude of $F_{\mathrm{b}}$ is about $0.1 \%$ of the total signal and is more sensitive to the background subtraction.) This slow frequency persists up to $30 \mathrm{~K}$ and its amplitude follows the usual LifshitzKosevich formula (Fig. 4b), with a small effective mass $m^{*}=0.45 \pm 0.1 m_{0}$, where $m_{0}$ is the free electron mass.

Using the $c$-axis resistance, we have measured the dependence of $F_{\mathrm{b}}$ on the angle $\theta$ at which the field is tilted away from the $c$ axis. In Fig. 4c, the oscillatory part of the $c$ axis resistance for $p=0.11$ at $T=15 \mathrm{~K}$ is plotted versus $1 / B \cos (\theta)$, and the angular dependence of $F_{\mathrm{b}}$ is displayed in Fig. $4 \mathrm{~d}$. $F_{\mathrm{b}}(\theta)$ varies approximately as $1 / \cos (\theta)$, indicating that the Fermi surface associated with $F_{\mathrm{b}}$ is a warped cylinder along the $c$ axis, as expected for a quasi-two-dimensional system.
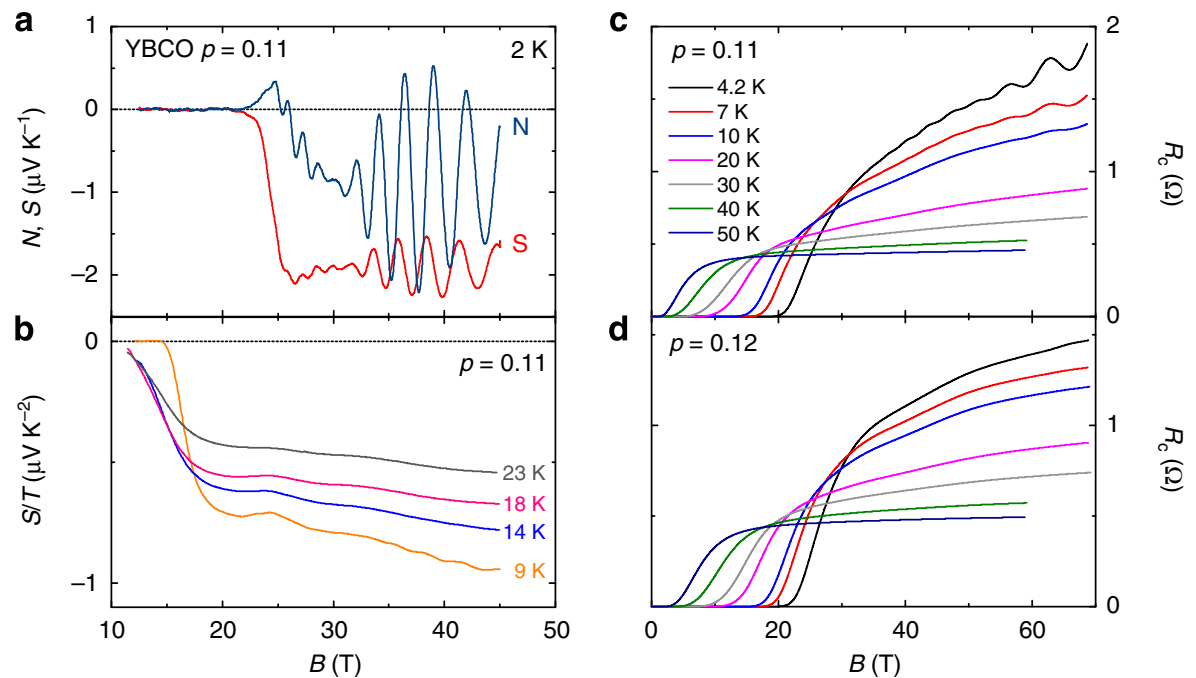

Figure 2 | Quantum oscillations in YBCO. (a) Seebeck ( $S$; red) and Nernst ( $N$; blue) signals in YBCO $p=0.11$ as a function of magnetic field $B$ at $T=2 \mathrm{~K}$. (b) Seebeck coefficient $S$, plotted as $S / T$ versus $B$, at temperatures as indicated. (c,d) $c$ axis electrical resistance $R_{\mathrm{c}}$ of $Y B C O$ samples with $p=0.11$ and $p=0.12$, as a function of $B$ up to $68 \mathrm{~T}$, at different temperatures as indicated. For all data, the field $B$ is along the $c$ axis.
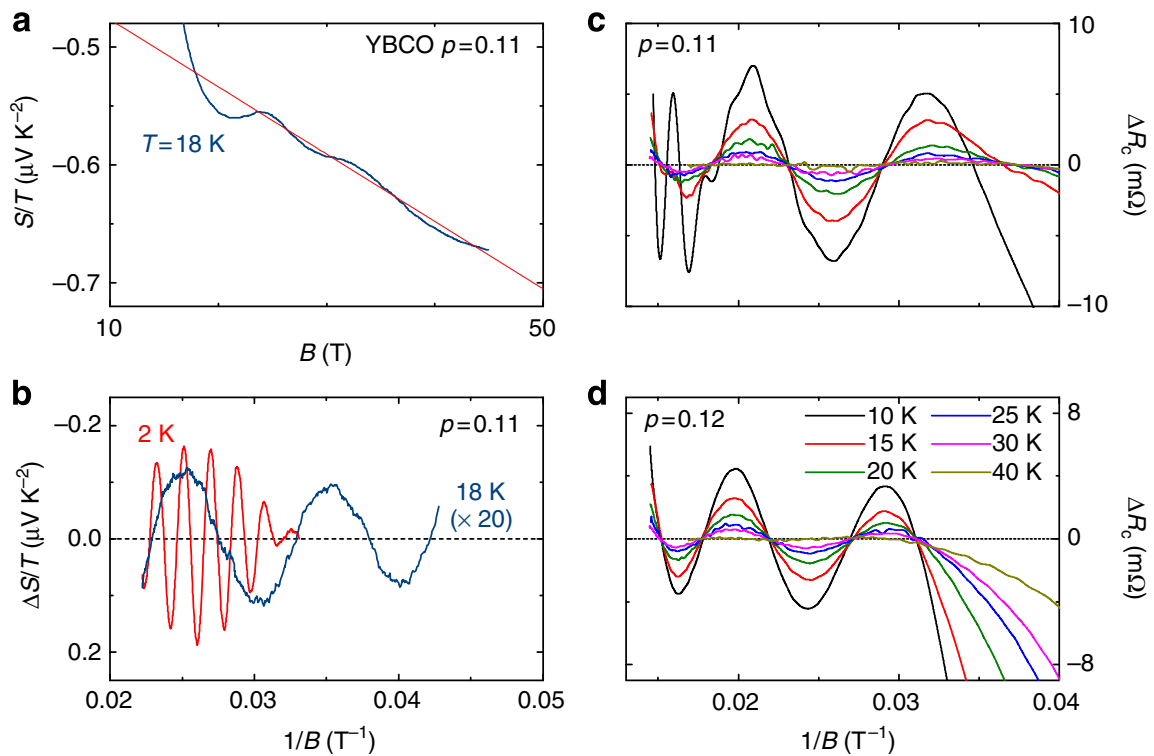

Figure 3 | Slow quantum oscillations. (a) Seebeck coefficient in YBCO $p=0.11$ (blue), plotted as $S / T$, as a function of field $B$ at $T=18 \mathrm{~K}$, showing slow oscillations about a linear background (red). (b) Oscillatory part of the Seebeck coefficient $\Delta S / T$ (obtained by subtracting a 2nd order polynomial from the raw data) as a function of $1 / B$, showing the usual fast quantum oscillations at $T=2 \mathrm{~K}$ (red), and the new slow oscillations with $F_{\mathrm{b}}=95 \pm 10 \mathrm{~T}$ at $T=18 \mathrm{~K}$ (blue, multiplied by 20). For clarity, the slow frequency $F_{\mathrm{b}}$ was removed from the data at $T=2 \mathrm{~K}$. (c,d) Oscillatory part of the $c$-axis electrical resistance $\Delta R_{\mathrm{c}}$ in YBCO (obtained by subtracting a third-order polynomial from the raw data) at $p=0.11$ and $p=0.12$ as a function of $1 / B$, at temperatures as indicated. The oscillations are periodic in $1 / B$, with a frequency $F_{\mathrm{b}}=95 \pm 10 \mathrm{~T}$ and $120 \pm 15 \mathrm{~T}$ at $p=0.11$ and 0.12 , respectively. 

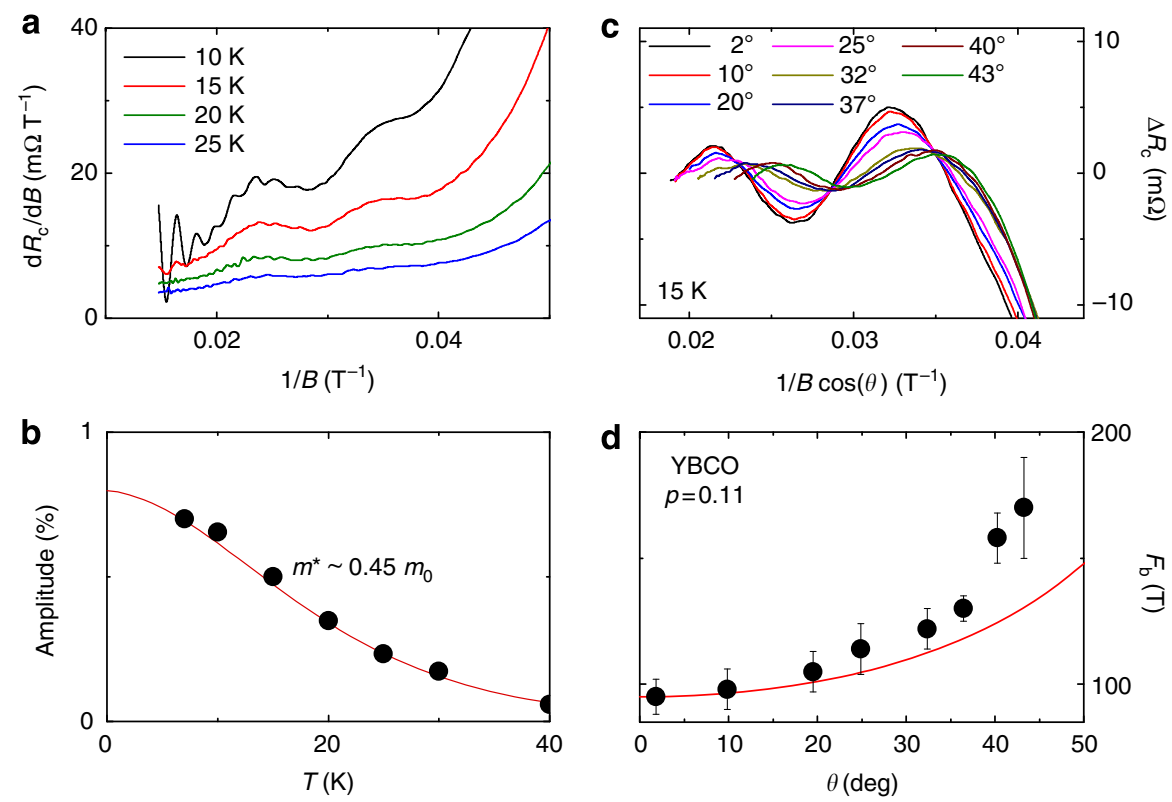

Figure 4 | Properties of the slow frequency at $\boldsymbol{p}=\mathbf{0 . 1 1}$. (a) Derivative of the $c$-axis resistance of sample $p=0.11$ with respect to field $B$, plotted versus $1 / B$ at temperatures as indicated. This confirms the presence of the slow frequency $F_{\mathrm{b}}$, irrespective of background subtraction. (b) Amplitude of $F_{\mathrm{b}}$ oscillations as a function of temperature (dots). The line is a Lifshitz-Kosevich fit to the data, giving an effective mass $m^{\star}=0.45 \pm 0.1 m_{0}$. (c) Oscillatory part of the $c$ axis resistance at different angles $\theta$ between the field and the $c$ axis, as indicated, as a function of $1 / B \cos (\theta)$ at $T=15 \mathrm{~K}$. A second-order polynomial background was subtracted from the raw data to extract $\Delta R_{\mathrm{c}}$. (d) Slow frequency $F_{\mathrm{b}}$ as a function of $\theta$ (dots). The red line is the function $1 / \cos (\theta)$. The error bars are a convolution of s.d. in the value of $F_{\mathrm{b}}$, for different fitting ranges and different orders of the polynomial background.

\section{Discussion}

The slow frequency $F_{\mathrm{b}} \sim 100 \mathrm{~T}$ reported here bears the key signatures of quantum oscillations and in the following discussion we argue that it comes from a small hole-like Fermi surface, distinct from the larger electron-like Fermi pocket responsible for the main frequency $F_{\mathrm{a} 1}=540 \mathrm{~T}$.

We note that the frequency $F_{\mathrm{b}}$ is nearly equal to the difference between the main frequency of the electron pocket $F_{\mathrm{a} 1}$ and its satellites $F_{\mathrm{a} 2}$ and $F_{\mathrm{a} 3}$. While the identification of the multiple $F_{\mathrm{a}}$ frequencies is not definitive, it is likely that two of them are associated with the two separate Fermi surfaces that come from the two $\mathrm{CuO}_{2}$ planes (bilayer) in the unit cell of YBCO. The third frequency could then either come from magnetic breakdown between these two Fermi surfaces ${ }^{27}$ or from a warping due to $c$-axis dispersion ${ }^{26,28}$. In layered quasi-two-dimensional materials, slow quantum oscillations can appear in the $c$-axis transport as a result of interlayer coupling ${ }^{29,30}$. Two observations allow us to rule out this scenario in the present context. First, $F_{\mathrm{b}}$ is observed in the in-plane Seebeck coefficient, which does not depend on the $c$-axis conductivity. Second, at a special field-angle $\theta$, called the Yamaji angle, where the $c$-axis velocity vanishes on average along a cyclotron orbit, one should see a vanishing $F_{\mathrm{b}}$. This is not seen in our field-angle dependence of $F_{b}$, which, if anything, only deviates upward from a cylindrical $1 / B \cos (\theta)$ dependence (Fig. 4d).

Quantum interference from magnetic breakdown between two bilayer-split orbits could in principle produce a difference frequency close to $F_{\mathrm{b}}$. In this scenario, however, the amplitudes of the two nearby frequencies $F_{\mathrm{a} 2}$ and $F_{\mathrm{a} 3}$ should be identical, irrespective of the field range, in disagreement with torque ${ }^{26}$ and $c$-axis resistance measurements (see Fig. 1d). Furthermore, in a magnetic breakdown scenario, we would expect $F_{\mathrm{b}}$ to scale with $F_{\mathrm{a}}$, since both frequencies originate from the same cyclotron orbits. This is not what we observe in our thermoelectric data: as seen in Fig. 2a, the amplitude of $F_{\mathrm{a}}$ is larger in the Nernst effect than in the Seebeck effect, yet $F_{\mathrm{b}}$ is only detected in the latter. This is strong evidence that $F_{\mathrm{a}}$ and $F_{\mathrm{b}}$ do not involve cyclotron orbits on the same Fermi surface. We therefore conclude that $F_{\mathrm{b}}$ must come from a distinct Fermi pocket, in contrast with the interpretation of ref. 31 in terms of quantum interference.

For a number of reasons, we infer that this second pocket in the reconstructed Fermi surface of YBCO is hole-like. The first reason is the strong dependence of resistivity $\rho$ and Hall coefficient $R_{\mathrm{H}}$ on magnetic field $B$, as observed in YBCO and in $\mathrm{YBa}_{2} \mathrm{Cu}_{4} \mathrm{O}_{8}$ (ref. 3), a closely related material with similar quantum oscillations ${ }^{32,33}$. For instance, $R_{\mathrm{H}}(B)$ goes from positive at low field to negative at high field ${ }^{3}$ and $\rho(B)$ exhibits a significant magnetoresistance ${ }^{25}$. These are natural consequences of having both electron and hole carriers. In $\mathrm{YBa}_{2} \mathrm{Cu}_{4} \mathrm{O}_{8}$, the Hall and resistivity data were successfully fit in detail to a two-band model of electrons and holes ${ }^{34}$.

A second indication that both the electron and hole carriers are present in underdoped YBCO is the fact that quantum oscillations are observed in the Hall coefficient ${ }^{35,36}$. In an isotropic single-band model, the Hall coefficient is simply given by $R_{\mathrm{H}}=1 / n e$, where $n$ is the carrier density and $e$ the electron charge. Quantum oscillations in $R_{\mathrm{H}}$ appear via the scattering rate, which enters $R_{\mathrm{H}}$ either when two or more bands of different mobility are present or when the scattering rate on a single band is strongly anisotropic. At low temperatures, however, where impurity scattering dominates, the latter scenario is improbable.

The most compelling evidence for the presence of hole-like carriers in underdoped YBCO comes from the magnitude of the Seebeck coefficient. In the $T=0$ limit and for a single band, it is given by ${ }^{22}$ :

$$
\frac{S}{T}= \pm \frac{\pi^{2}}{3} \frac{k_{\mathrm{B}}}{e} \frac{1}{T_{\mathrm{F}}}\left(\frac{3}{2}+\zeta\right)
$$


where $k_{\mathrm{B}}$ is Boltzmann's constant, $T_{\mathrm{F}}$ is the Fermi temperature and $\zeta=0$ or $-1 / 2$ depending on whether the relaxation time or the mean free path is assumed to be energy independent, respectively. The sign of $S / T$ depends on whether the carriers are holes $(+)$ or electrons $(-)$. This expression has been found to work very well in a variety of correlated electron metals ${ }^{22}$. We stress that $S / T$ (in the $T=0$ limit) is governed solely by $T_{\mathrm{F}}$, which allows a direct quantitative comparison with quantum oscillation data, with no assumption on pocket multiplicity. This contrasts with the specific heat, which depends on the number of Fermi pockets (see below).

In Fig. 5a, we reproduce normal-state Seebeck data in YBCO at four dopings, plotted as $S / T$ versus $T$ (from ref. 7). $S / T$ goes from positive at high $T$ to negative at low $T$, in agreement with a similar sign change in $R_{\mathrm{H}}(T)$ (refs 3,4 ), both evidence that the dominant carriers at low $T$ are electron like. Extrapolating $S / T$ to $T=0$ as shown by the dashed lines in Fig. $5 \mathrm{a}$, we obtain the residual values and plot them as a function of doping in Fig. 5b ( $S_{\text {measured }}$, red squares). We see that the size of the residual term is largest (that is, is most strongly negative) at $p=0.11$, and that it decreases on both sides.

This doping-dependent $S / T$ is to be compared with the Fermi temperature directly measured by quantum oscillations via:

$$
T_{\mathrm{F}}=\frac{e \hbar}{k_{\mathrm{B}}} \frac{F}{m^{*}}
$$

where $F$ is the frequency, $m^{*}$ the effective mass and assuming a parabolic dispersion. For YBCO at $p=0.11$, the electron pocket gives $F_{\mathrm{a} 1}=540 \pm 20 \mathrm{~T}$ and $m^{\star}=1.76 m_{0}$, so that $T_{\mathrm{F}}=410 \pm 20 \mathrm{~K}$ and hence $S_{\mathrm{e}} / T=-1.0 \mu \mathrm{V} \mathrm{K}^{-2}\left(-0.7 \mu \mathrm{V} \mathrm{K}^{-2}\right)$, for $\zeta=0(-1 / 2)$. In Fig. 5a, the measured $S / T$ extrapolated to $T \rightarrow 0$ gives a value of $-0.9 \mu \mathrm{VK}^{-2}$. The electron pocket alone therefore accounts by itself for essentially the entire measured Seebeck signal at $p=0.11$. From quantum oscillation measurements at different dopings ${ }^{24,37,38}$, we know the values of $F_{\mathrm{a} 1}$ and $m^{*}$ from $p=0.09$ to $p=0.13$, and can therefore determine the evolution of $S_{\mathrm{e}} / T$ in that doping interval. The result is plotted as blue dots in Fig. 5b, where we see that the calculated $\left|S_{\mathrm{e}} / T\right|$ increases by a factor 2.5 between $p=0.11$ and $p=0.09$. This is because the mass $m^{*}$ increases strongly as $p \rightarrow 0.08$ (ref. 38), while $F_{\mathrm{a} 1}$ decreases only slightly ${ }^{18}$. This strong increase in the calculated $\left|S_{\mathrm{e}} / T\right|$ is in stark contrast with the measured value of $|S / T|$, which decreases by a factor of 3 between $p=0.11$ and $p=0.09$ (Fig. 5b). To account for the observed doping dependence of the thermopower in YBCO, we are led to conclude that there must be a hole-like contribution to $S / T$. We emphasize that the Hall coefficient $R_{\mathrm{H}}$ (ref. 4) measured well above $B_{\mathrm{c} 2}$ (ref. 21) displays the same dome-like dependence on doping as the Seebeck coefficient ${ }^{7}$, which further confirms the presence of a hole-like Fermi pocket.

In a two-band model, the total Seebeck coefficient is given by

$$
S=\frac{S_{\mathrm{e}} \sigma_{\mathrm{e}}+S_{\mathrm{h}} \sigma_{\mathrm{h}}}{\sigma_{\mathrm{e}}+\sigma_{\mathrm{h}}}
$$

where the hole $(h)$ and electron $(e)$ contributions are weighted by their respective conductivities $\sigma_{\mathrm{h}}$ and $\sigma_{\mathrm{e}}$. As shown in Fig. 5c, we can account for the measured $S / T$ at $T \rightarrow 0$ by adding a hole-like contribution, $S_{\mathrm{h}}$, which we estimate from $F_{\mathrm{b}}=95 \pm 10 \mathrm{~T}$ and $m^{*}=0.45 m_{0}$, giving $T_{\mathrm{F}}=280 \pm 80 \mathrm{~K}$. Assuming for simplicity that $S_{\mathrm{h}}$ is doping independent leaves the ratio of conductivities, $\sigma_{\mathrm{e}} / \sigma_{\mathrm{h}}$, as the only adjustable parameter in the above two-band expression (equation (3)). In Fig. 5d, we plot the resulting $\sigma_{\mathrm{e}} / \sigma_{\mathrm{h}}$ as a function of doping, and we see that it peaks at $p=0.11$ and drops on either side. This is consistent with the fact that the amplitude of the fast quantum oscillations is largest at $p=0.11$, and much smaller away from that doping ${ }^{18}$, direct evidence that
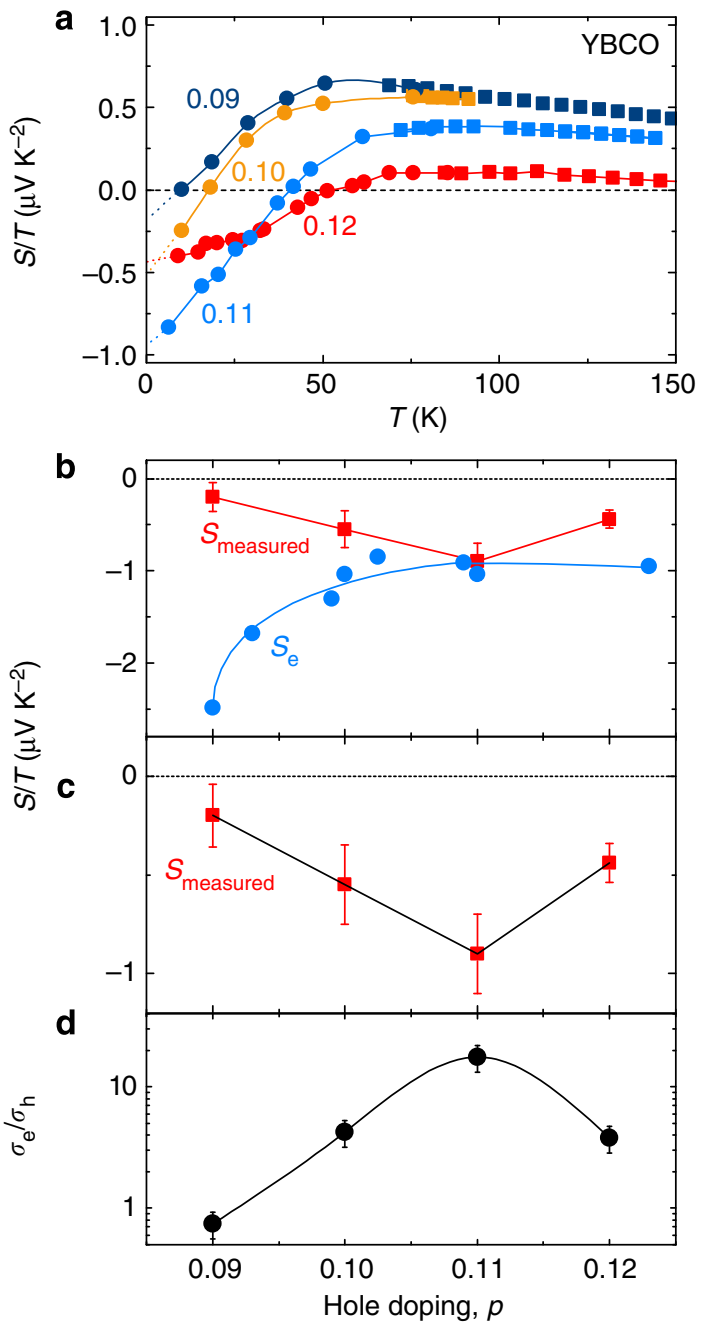

Figure 5 | Evidence of a hole-like contribution to the Seebeck coefficient. (a) Normal-state Seebeck coefficient $S / T$ as a function of temperature for YBCO at four dopings, as indicated (adapted from ref. 7). The squares and dots are data in zero field and in $28 \mathrm{~T}$, respectively. The dotted lines are extrapolations of $S / T$ to $T \rightarrow 0$, whose values are plotted in $\mathbf{b}, \mathbf{c}$ (red squares). The lines are a guide to the eye. (b) Extrapolated value of $S / T$ at $T=0$ as a function of doping $\left(S_{\text {measured, }}\right.$ red squares; from data and extrapolations in $\mathbf{a})$. The error bars represent the uncertainty in extrapolating to $T=0$. The blue dots $\left(S_{e}\right)$ indicate $S / T(T \rightarrow 0)$ for the electron pocket alone, calculated from the main quantum oscillation frequency $F_{\mathrm{a} 1}$ and mass $m^{\star}$ (from refs 24,37,38) (using equations (1) and (2)). The blue line is a guide to the eye. (c) The red squares $\left(S_{\text {measured }}\right)$ are identical to those in $\mathbf{b}$. Using a two-band model (equation (3)), we include the contribution of the hole pocket $\left(S_{h}\right)$ using the measured Fermi temperature associated with the slow frequency $F_{\mathrm{b}}\left(T_{\mathrm{F}}=280 \pm 80 \mathrm{~K}\right.$; see text). With the conductivity ratio $\sigma_{\mathrm{e}} / \sigma_{\mathrm{h}}$ as the only fit parameter, this model (black line) reproduces the measured data (red squares). (d) Ratio of electron-to-hole conductivities, $\sigma_{\mathrm{e}} / \sigma_{\mathrm{h}}$ (black circles), from a fit (black line, c) to the measured values of $S / T$ at $T=0$ (red squares, $\mathbf{b}, \mathbf{c}$ ). The black line is a guide to the eye. The error bars are derived from the errors on $S_{\text {measured. }}$

the mobility of the electron pocket is maximal at $p=0.11$. This is clearly seen in the resistance data in Fig. $2 c, d$, where the amplitude of the quantum oscillations of the electron pocket is strongly reduced when going from $p=0.11$ to 0.12 . In contrast, Fig. 3c,d shows that the amplitude of the oscillations from the hole pocket remains nearly constant: at $T=4.2 \mathrm{~K}$ and $H=68 \mathrm{~T}$, their relative amplitude is $\Delta R_{\mathrm{c}} / R_{\mathrm{c}}=0.036 \%$ at $p=0.11$ and $0.03 \%$ 
at $p=0.12$. The change in conductivity ratio therefore comes mostly from a change in $\sigma_{\mathrm{e}}$.

To summarize, in addition to the two-band description of transport data in $\mathrm{YBa}_{2} \mathrm{Cu}_{4} \mathrm{O}_{8}$ (ref. 34), the doping dependence of the Seebeck ${ }^{7}$ and Hall ${ }^{4}$ coefficients in YBCO is firm evidence that the reconstructed Fermi surface of underdoped YBCO (for $0.08<p<0.18$ ) contains not only the well-established electron pocket $^{4}$, but also another hole-like surface (of lower mobility). We combine this evidence with our discovery of an additional small Fermi surface to conclude that this new pocket is hole like.

From the measured effective mass $m^{*}$, the residual linear term $\gamma$ in the electronic specific heat $C_{\mathrm{e}}(T)$ at $T \rightarrow 0$ can be estimated through the relation ${ }^{39}$

$$
\gamma=\left(1.46 \mathrm{mJK}^{-2} \mathrm{~mol}\right) \sum_{\mathrm{i}}\left(n_{\mathrm{i}} m_{\mathrm{i}}^{*} / m_{0}\right)
$$

where $n_{\mathrm{i}}$ is the multiplicity of the $\mathrm{i}^{\text {th }}$ type of pocket in the first Brillouin zone (this expression assumes an isotropic Fermi liquid in two dimensions with a parabolic dispersion). For a Fermi surface containing one electron pocket and two hole pockets per $\mathrm{CuO}_{2}$ plane, we obtain a total mass of $(1.7 \pm 0.2)+2$ $(0.45 \pm 0.1)=2.6 \pm 0.4 m_{0}$, giving $\gamma=7.6 \pm 0.8 \mathrm{~mJ} \mathrm{~K}^{-2} \mathrm{~mol}$ (for two $\mathrm{CuO}_{2}$ planes per unit cell). High-field measurements of $C_{\mathrm{e}}$ at $T \rightarrow 0$ in YBCO at $p \sim 0.1$ yield $\gamma=5 \pm 1 \mathrm{~mJ} \mathrm{~K}^{-2} \mathrm{~mol}$ (ref. 39) at $B>B_{\mathrm{c} 2}=30 \mathrm{~T}$ (ref. 21). We therefore find that the Fermi surface of YBCO can contain at most two of the small hole pockets reported here, in addition to only one electron pocket. No further sheet can realistically be present in the Fermi surface.

There is compelling evidence that the Fermi surface of YBCO is reconstructed by the CDW order detected by NMR and X-ray diffraction. In particular, Fermi-surface reconstruction ${ }^{4,7}$ and CDW modulations ${ }^{19,20}$ are detected in precisely the same region of the temperature-doping phase diagram. Because the CDW modulations are along both the $a$ and $b$ axes, the reconstruction naturally produces a small closed electron pocket along the Brillouin zone diagonal, at the so-called nodal position ${ }^{40,41}$. Given the wavevectors measured by X-ray diffraction, there will also be small closed hole-like ellipses located between the diamondshaped nodal electron pockets. An example of the Fermi surface calculated $^{42}$ using the measured CDW wavevectors is sketched in Fig. 1c. It contains two distinct closed pockets: a nodal electron pocket of area such that $F_{\mathrm{e}} \sim 430 \mathrm{~T}$ and a hole-like ellipse such that $F_{\mathrm{h}} \sim 90 \mathrm{~T} p=0.11$ (ref. 42 ). Note that a reconstruction by a commensurate wavevector $q=1 / 3 \pi / a$, very close to the measured value, yields one electron and two hole pockets per Brillouin zone, as assumed in our calculation of $\gamma$ above.

If, as indeed observed in YBCO at $p=0.11$ (ref. 20), the CDW modulations are anisotropic in the $a-b$ plane, the ellipse pointing along the $a$ axis will be different from that pointing along the $b$ axis $^{42}$. If one of the ellipses is close enough to the electron pocket, that is, if the gap between the two is small enough, magnetic breakdown will occur between the hole and the electron pockets, and this could explain the complex spectra of multiple quantum oscillations seen in underdoped YBCO (Fig. 1d).

In most models of Fermi-surface reconstruction by CDW order, the size of the Fermi pockets can be made to agree with experiments using a reasonable set of parameters. For example, a similar Fermi surface (with one electron pocket and two hole pockets) is also obtained if one considers a 'criss-crossed' stripe pattern instead of a checkerboard ${ }^{43}$. At this level, there is consistency between our quantum oscillation measurements and models of Fermi-surface reconstruction by the CDW order. However, in addition to the electron and hole pockets, the folding of the large Fermi surface produces other segments of Fermi surface whose total contribution to $\gamma$ greatly exceeds that allowed by the specific heat data. Consequently, there must exist a mechanism that removes parts of the Fermi surface beyond the reconstruction by the $\mathrm{CDW}$ order. A possible mechanism is the pseudogap. The loss of the antinodal states caused by the pseudogap would certainly remove parts of the reconstructed Fermi surface.

Further theoretical investigations are needed to understand how pseudogap and CDW order are intertwined in underdoped cuprates. An important point in this respect is the fact that, unlike in $\mathrm{Bi}_{2} \mathrm{Sr}_{2}-{ }_{x} \mathrm{La}_{x} \mathrm{CuO}_{6+\mathrm{d}}$ (ref. 44), the $\mathrm{CDW}$ wavevector measured by X-ray diffraction in YBCO and in Hg1201 (ref. 14) does not connect the hot spots where the large Fermi surface intersects the antiferromagnetic Brillouin zone (Fig. 1c), nor does it nest the flat antinodal parts of that large Fermi surface (Fig. 1c).

\section{Methods}

Samples. Single crystals of YBCO with $y=6.54,6.62$ and 6.67 were obtained by flux growth at $\mathrm{UBC}^{45}$. The superconducting transition temperature $T_{\mathrm{c}}$ was determined as the temperature below which the zero-field resistance $R=0$. The hole doping $p$ is obtained from $T_{c}$ (ref. 46), giving $p=0.11$ for $y=6.54$ and 6.62 , and $p=0.12$ for $y=6.67$. The samples with $y=6.54$ and 6.62 have a high degree of ortho-II oxygen order, and the sample with $y=6.67$ has ortho-VIII order. The samples are detwinned rectangular platelets, with the $a$ axis parallel to the length (longest dimension) and the $b$ axis parallel to the width. The electrical contacts are diffused evaporated gold pads with a contact resistance less than $1 \Omega$.

Thermoelectric measurements. The thermoelectric response of YBCO with $y=6.54(p=0.11)$ was measured at the National High Magnetic Field Laboratory (NHMFL) in Tallahassee, Florida, up to $45 \mathrm{~T}$, in the temperature range from 2 to $40 \mathrm{~K}$. The Seebeck and Nernst coefficients are given by $S \equiv-\nabla V_{x} / \nabla T_{x}$ and $v \equiv N / B \equiv\left(\nabla V_{y} / \nabla T_{x}\right) / B$, respectively, where $\nabla V_{x}\left(\nabla V_{y}\right)$ is the longitudinal (transverse) voltage gradient caused by a temperature gradient $\nabla T_{x}$, in a magnetic field B ||z. A constant heat current was sent along the $a$ axis of the single crystal, generating a temperature difference $\Delta T_{x}$ across the sample. $\Delta T_{x}$ was measured with two uncalibrated Cernox chip thermometers (Lakeshore), referenced to a third, calibrated Cernox. The longitudinal and transverse electric fields were measured using nanovolt preamplifiers and nanovoltmeters. All measurements were performed with the temperature of the experiment stabilized within $\pm 10 \mathrm{mK}$ and the magnetic field $B$ swept at a constant rate of $0.4-0.9 \mathrm{~T} \mathrm{~min}^{-1}$ between positive and negative maximal values, with the heat on. The field was applied normal to the $\mathrm{CuO}_{2}$ planes $(B\|z\| c)$.

Since the Seebeck coefficient $S$ is symmetric with respect to the magnetic field, it is obtained by taking the mean value between positive and negative fields:

$$
S=E_{x} /(\partial T / \partial x)=\left[\Delta V_{x}(B)+\Delta V_{x}(-B)\right] /\left(2 \Delta T_{x}\right),
$$

where $\Delta V_{x}$ is the difference in the voltage along $x$ measured with and without thermal gradient. This procedure removes any transverse contribution that could appear due to slightly misaligned contacts. The longitudinal voltages and the thermal gradient being measured on the same pair of contacts, no geometric factor is involved.

The Nernst coefficient $N$ is antisymmetric with respect to the magnetic field; therefore, it is obtained by the difference:

$$
N=E_{y} /(\partial T / \partial x)=(L / w)\left[V_{y}(B)-V_{y}(-B)\right] /\left(2 \Delta T_{x}\right),
$$

where $L$ and $w$ are the length and width of the sample, respectively, along $x$ and $y$ and $V_{y}$ is the voltage along $y$ measured with the heat current on. This antisymmetrization procedure removes any longitudinal thermoelectric contribution and a constant background from the measurement circuit. The uncertainty on $N$ comes from the uncertainty in determining $L$ and $w$, giving typically an error bar of $\pm 10 \%$.

Resistance measurements. The $c$-axis resistance was measured at the Laboratoire National des Champs Magnétiques Intenses (LNCMI) in Toulouse, France, in pulsed magnetic fields up to $68 \mathrm{~T}$. Measurements were performed in a conventional four-point configuration, with a current excitation of $5 \mathrm{~mA}$ at a frequency of $\sim 60 \mathrm{kHz}$. Electrical contacts to the sample were made with large current pads and small voltage pads mounted across the top and bottom so as to short out any inplane current. A high-speed acquisition system was used to digitize the reference signal (current) and the voltage drop across the sample at a frequency of $500 \mathrm{kHz}$ The data were analysed with software that performs the phase comparison. $\theta$ is the angle between the magnetic field and the $c$ axis, and measurements were done at $\theta=0^{\circ}$ up to $68 \mathrm{~T}$ and at various angles $\theta$ up to $58 \mathrm{~T}$. The uncertainty on the absolute value of the angle is about $1^{\circ}$. 


\section{References}

1. Doiron-Leyraud, N. et al. Quantum oscillations and the Fermi surface in an underdoped high- $T_{\mathrm{c}}$ superconductor. Nature 447, 565-568 (2007).

2. Barišić, N. et al. Universal quantum oscillations in the underdoped cuprate superconductors. Nat. Phys. 9, 761-764 (2013).

3. LeBoeuf, D. et al. Electron pockets in the Fermi surface of hole-doped high- $T_{\mathrm{c}}$ superconductors. Nature 450, 533-536 (2007).

4. LeBoeuf, D. et al. Lifshitz critical point in the cuprate superconductor $\mathrm{YBa}_{2} \mathrm{Cu}_{3} \mathrm{O}_{\mathrm{y}}$ from high-field Hall effect measurements. Phys. Rev. B 83, 054506 (2011)

5. Doiron-Leyraud, N. et al. Hall, Seebeck, and Nernst coefficients of underdoped $\mathrm{HgBa}_{2} \mathrm{CuO}_{4+\delta}$ : Fermi-surface reconstruction in an archetypal cuprate superconductor. Phys. Rev. X 3, 021019 (2013).

6. Chang, J. et al. Nernst and Seebeck coefficients of the cuprate superconductor $\mathrm{YBa}_{2} \mathrm{Cu}_{3} \mathrm{O}_{6.67}$ : a study of Fermi surface reconstruction. Phys. Rev. Lett. 104, 057005 (2010)

7. Laliberté, F. et al. Fermi-surface reconstruction by stripe order in cuprate superconductors. Nat. Commun. 2, 432 (2011).

8. Taillefer, L. et al. Fermi-surface reconstruction in high- $T_{\mathrm{c}}$ superconductors. J. Phys.: Condens. Matter 21, 164212 (2009).

9. Fink, J. et al. Phase diagram of charge order in $\mathrm{La}_{1.8-\mathrm{x}} \mathrm{Eu}_{0.2} \mathrm{Sr}_{\mathrm{x}} \mathrm{CuO}_{4}$ from resonant soft x-ray diffraction. Phys. Rev. B 83, 092503 (2011).

10. Wu, T. et al. Magnetic-field-induced charge-stripe order in the hightemperature superconductor $\mathrm{YBa}_{2} \mathrm{Cu}_{3} \mathrm{O}_{\mathrm{y}}$. Nature 477, 191-194 (2011).

11. Ghiringhelli, G. et al. Long-range incommensurate charge fluctuations in ( $\mathrm{Y}, \mathrm{Nd}) \mathrm{Ba}_{2} \mathrm{Cu}_{3} \mathrm{O}_{6+\mathrm{x}}$. Science 337, 821-825 (2012).

12. Chang, J. et al. Direct observation of competition between superconductivity and charge density wave order in $\mathrm{YBa}_{2} \mathrm{Cu}_{3} \mathrm{O}_{6.67}$. Nat. Phys. 8, 871-876 (2012).

13. Achkar, A. J. et al. Distinct charge orders in the planes and chains of ortho-IIIordered $\mathrm{YBa}_{2} \mathrm{Cu}_{3} \mathrm{O}_{6+\delta}$ superconductors identified by resonant elastic $\mathrm{x}$-ray scattering. Phys. Rev. Lett. 109, 167001 (2012).

14. Tabis, W. et al. Charge order and its connection with Fermi-liquid charge transport in a pristine high- $T_{\mathrm{c}}$ cuprate. Nat. Commun. 5, 5875 (2014).

15. LeBoeuf, D. et al. Thermodynamic phase diagram of static charge order in underdoped $\mathrm{YBa}_{2} \mathrm{Cu}_{3} \mathrm{O}_{\mathrm{y}}$. Nat. Phys. 9, 79-83 (2013).

16. Hoffman, J. E. et al. A four unit cell periodic pattern of quasi-particle states surrounding vortex cores in $\mathrm{Bi}_{2} \mathrm{Sr}_{2} \mathrm{CaCu}_{2} \mathrm{O}_{8+\delta}$. Science 295, 466-469 (2002).

17. Kohsaka, Y. et al. An intrinsic bond-centered electronic glass with unidirectional domains in underdoped cuprates. Science 315, 1380-1385 (2007).

18. Vignolle, B., Vignolles, D., Julien, M.-H. \& Proust, C. From quantum oscillations to charge order in high- $T_{\mathrm{c}}$ copper oxides in high magnetic fields C. R. Phys. 14, 39-52 (2013).

19. Hücker, M. et al. Competing charge, spin, and superconducting orders in underdoped $\mathrm{YBa}_{2} \mathrm{Cu}_{3} \mathrm{O}_{\mathrm{y}}$. Phys. Rev. B 90, 054514 (2014).

20. Blanco-Canosa, S. et al. Resonant x-ray scattering study of charge-density wave correlations in $\mathrm{YBa}_{2} \mathrm{Cu}_{3} \mathrm{O}_{6+\mathrm{x}}$. Phys. Rev. B 90, 054513 (2014).

21. Grissonnanche, G. et al. Direct measurement of the upper critical field in cuprate superconductors. Nat. Commun. 5, 3280 (2014).

22. Behnia, K., Jaccard, D. \& Flouquet, J. On the thermoelectricity of correlated electrons in the zero-temperature limit. J. Phys.: Condens. Matter 16, 5187 (2004).

23. Behnia, K. The Nernst effect and the boundaries of the Fermi liquid picture. J. Phys.: Condens. Matter 21, 113101 (2009).

24. Ramshaw, B. J. et al. Angle dependence of quantum oscillations in $\mathrm{YBa}_{2} \mathrm{Cu}_{3} \mathrm{O}_{6.59}$ shows free-spin behaviour of quasiparticles. Nat. Phys. 7, 234-238 (2010).

25 . Vignolle, B. et al. Coherent $c$-axis transport in the underdoped cuprate superconductor $\mathrm{YBa}_{2} \mathrm{Cu}_{3} \mathrm{O}_{y}$. Phys. Rev. B 85, 224524 (2012).

26. Audouard, A. et al. Multiple quantum oscillations in the de Haas-van Alphen spectra of the underdoped high-temperature superconductor $\mathrm{YBa}_{2} \mathrm{Cu}_{3} \mathrm{O}_{6.5}$. Phys. Rev. Lett. 103, 157003 (2009).

27. Sebastian, S. E. et al. Quantum oscillations from nodal bilayer magnetic breakdown in the underdoped high temperature superconductor $\mathrm{YBa}_{2} \mathrm{Cu}_{3} \mathrm{O}_{6+\mathrm{x}}$. Phys. Rev. Lett. 108, 196403 (2012).

28. Sebastian, S. E. et al. Fermi-liquid behavior in an underdoped high- $T_{c}$ superconductor. Phys. Rev. B 81, 140505 (2010).

29. Grigoriev, P. et al. Anomalous beating phase of the oscillating interlayer magnetoresistance in layered metals. Phys. Rev. B 65, 060403(R) (2002).

30. Kartsovnik, M. et al. Slow oscillations of magnetoresistance in quasi-twodimensional metals. Phys. Rev. Lett. 89, 126802 (2002).

31. Sebastian, S. E. et al. Normal-state nodal electronic structure in underdoped high- $T_{\mathrm{c}}$ copper oxides. Nature 511, 61-64 (2014).

32. Yelland, E. A. et al. Quantum oscillations in the underdoped cuprate $\mathrm{YBa}_{2} \mathrm{Cu}_{4} \mathrm{O}_{8}$. Phys. Rev. Lett. 100, 047003 (2008).

33. Bangura, A. F. et al. Small Fermi surface pockets in underdoped high temperature superconductors: observation of Shubnikov-de Haas oscillations in $\mathrm{YBa}_{2} \mathrm{Cu}_{4} \mathrm{O}_{8}$. Phys. Rev. Lett. 100, 047004 (2008).
34. Rourke, P. M. C. et al. Fermi-surface reconstruction and two-carrier model for the Hall effect in $\mathrm{YBa}_{2} \mathrm{Cu}_{4} \mathrm{O}_{8}$. Phys. Rev. B 82, 020514 (2010).

35. Chakravarty, S. \& Kee, H.-Y. Fermi pockets and quantum oscillations of the Hall coefficient in high-temperature superconductors. Proc. Natl Acad. Sci. USA 105, 8835-8839 (2008)

36. Kikugawa, N., Rost, A.W., Hicks, C. W., Schofield, A. J. \& Mackenzie, A. P. $\mathrm{Ca}_{3} \mathrm{Ru}_{2} \mathrm{O}_{7}$ : density wave formation and quantum oscillations in the Hall resistivity. J. Phys. Soc. Jpn 79, 024704 (2010).

37. Jaudet, C. et al. de Haas-van Alphen oscillations in the underdoped high-temperature superconductor $\mathrm{YBa}_{2} \mathrm{Cu}_{3} \mathrm{O}_{6.5}$. Phys. Rev. Lett. 100, 187005 (2008).

38. Sebastian, S. E. et al. Metal-insulator quantum critical point beneath the high- $T_{\mathrm{c}}$ superconducting dome. Proc. Natl Acad. Sci. USA 107, 6175-6179 (2010).

39. Riggs, S. C. et al. Heat capacity through the magnetic-field-induced resistive transition in an underdoped high-temperature superconductor. Nat. Phys. 7, 332-335 (2011).

40. Harrison, N. \& Sebastian, S. E. Protected nodal electron pocket from multiple$Q$ ordering in underdoped high temperature superconductors. Phys. Rev. Lett. 106, 226402 (2011).

41. Sebastian, S. E., Harrison, N. \& Lonzarich, G. G. Towards resolution of the Fermi surface in underdoped high- $T_{\mathrm{c}}$ superconductors. Rep. Prog. Phys. 75, 102501 (2012)

42. Allais, A., Chowdhury, D. \& Sachdev, S. Connecting high-field quantum oscillations to the pseudogap in the underdoped cuprates. Nat. Commun. 5, 5771 (2014).

43. Maharaj, A. V., Hosur, P. \& Raghu, S. Crisscrossed stripe order from interlayer tunneling in hole-doped cuprates. Phys. Rev. B 90, 125108 (2014).

44. Comin, R. et al. Charge order driven by Fermi-arc instability in $\mathrm{Bi}_{2} \mathrm{Sr}_{2-x} \mathrm{La}_{\mathrm{x}} \mathrm{CuO}_{6+\delta}$. Science 343, 390-392 (2014).

45. Liang, R., Bonn, D. A. \& Hardy, W. N. Growth of YBCO single crystals by the self-flux technique. Phil. Mag. 92, 2563 (2012).

46. Liang, R., Bonn, D. A. \& Hardy, W. N. Evaluation of $\mathrm{CuO}_{2}$ plane hole doping in $\mathrm{YBa}_{2} \mathrm{Cu}_{3} \mathrm{O}_{6+\mathrm{x}}$ single crystals. Phys. Rev. B 73, 180505 (2006).

\section{Acknowledgements}

We thank A. Allais, K. Behnia, A. Carrington, S. Chakravarty, B. Fauqué, A. Georges, N. E. Hussey, M.-H. Julien, S.A. Kivelson, M.R. Norman, S. Raghu, S. Sachdev, A.-M. Tremblay and C. Varma for fruitful discussions. We thank J. Béard and P. Frings for their assistance with the experiments at the LNCMI. The work in Toulouse was supported by the French ANR SUPERFIELD, the EMFL and the LABEX NEXT. A portion of this work was performed at the National High Magnetic Field Laboratory, which is supported by the National Science Foundation Cooperative Agreement No. DMR-1157490, the State of Florida, and the U.S. Department of Energy. R.L., D.A.B. and W.N.H. acknowledge support from NSERC. L.T. acknowledges support from the Canadian Institute for Advanced Research and funding from NSERC, FRQNT, the Canada Foundation for Innovation and a Canada Research Chair.

\section{Author contributions}

N.D.-L., S.R.d.C. and J.-H.P. performed the Seebeck and Nernst measurements at the NHMFL in Tallahassee. N.D.-L., F.L. and E.H. analysed the Seebeck data. S.B., S.L., D.L.B., D.V., B.V. and C.P. performed and analysed the resistance measurements at the LNCMI in Toulouse. B.J.R., R.L., D.A.B. and W.N.H. prepared the YBCO single crystals at UBC (crystal growth, annealing, detwinning and contacts). L.T. and N.D.-L. supervised the thermoelectric measurements. C.P. supervised the pulsed-field measurements. N.D.-L., L.T. and C.P. wrote the manuscript with input from all authors.

\section{Additional information}

Competing financial interests: The authors declare no competing financial interests.

Reprints and permission information is available online at http://npg.nature.com/ reprintsandpermissions/

How to cite this article: Doiron-Leyraud, N. et al. Evidence for a small hole pocket in the Fermi surface of underdoped $\mathrm{YBa}_{2} \mathrm{Cu}_{3} \mathrm{O}_{y}$. Nat. Commun. 6:6034 doi: $10.1038 /$ ncomms7034 (2015).

This work is licensed under a Creative Commons Attribution 4.0 International License. The images or other third party material in this article are included in the article's Creative Commons license, unless indicated otherwise in the credit line; if the material is not included under the Creative Commons license, users will need to obtain permission from the license holder to reproduce the material To view a copy of this license, visit http://creativecommons.org/licenses/by/4.0/ 